\title{
STRUCTURES OF CHROMOSPHERIC MAGNETIC FIELDS IN ACTIVE REGIONS
}

\author{
HONGQI ZHANG, GUOXIANG AI, WEI LI AND JIMIN CHEN \\ Beijing Astronomical Observatory, Chinese Academy of Sciences, \\ Beijing 100080, China
}

ABSTRACT In this paper, we study the distribution of chromospheric magnetic fields and analyze reversed polarity structures on chromospheric magnetograms in active regions.

\section{INTRODUCTION}

Chromospheric magnetograms can be obtained with the hydrogen $\mathrm{H} \beta \lambda 4861.34$ $\AA$ line in addition to photospheric vector magnetograms using the video vector magnetograph at Huairou Solar Observing Station of Beijing Observatory. The bandpass of the birefringent filter with 3 sets of KDP crystal modulators is about $1 / 8 \AA$. The working wavelength of the filter for the measurement of chromospheric fields can be changed to (a) $-0.24 \AA$ away from the center of $\mathrm{H} \beta$ $\lambda 4861.34 \AA$ (general case) or (b) various wavelengths near the $\mathrm{H} \beta$ line center.

\section{FINE STRUCTURES OF THE CHROMOSPHERIC MAGNETIC FIELD}

The fine structures of the chromospheric magnetic field and their relation with the photospheric field were described by Zhang et al. (1991). The main results are the following:

(a) The chromospheric superpenumbral magnetic field, measured with the $\mathrm{H} \beta$ line, presents a spoke-like structure. It consists of thick magnetic fibrils.

(b) Polarity differences between the chromospheric and photospheric magnetic structures can be found near magnetic neutral lines.

(c) The distribution of the chromospheric network magnetic field is similar to that of the photosphere. The photospheric network field extends to the chromosphere as magnetic fibrils.

(d) Polarity reversals between the photosphere and chromosphere are probably caused by twisted magnetic lines of force in the lower solar atmosphere. The characteristic scale of this kind of polarity reversal should be smaller than the thickness of the chromosphere due to the limitation of the geometric figure of the flux ropes.

(e) Some chromospheric magnetic features show faster variations than the photospheric field (Zhang 1991). 


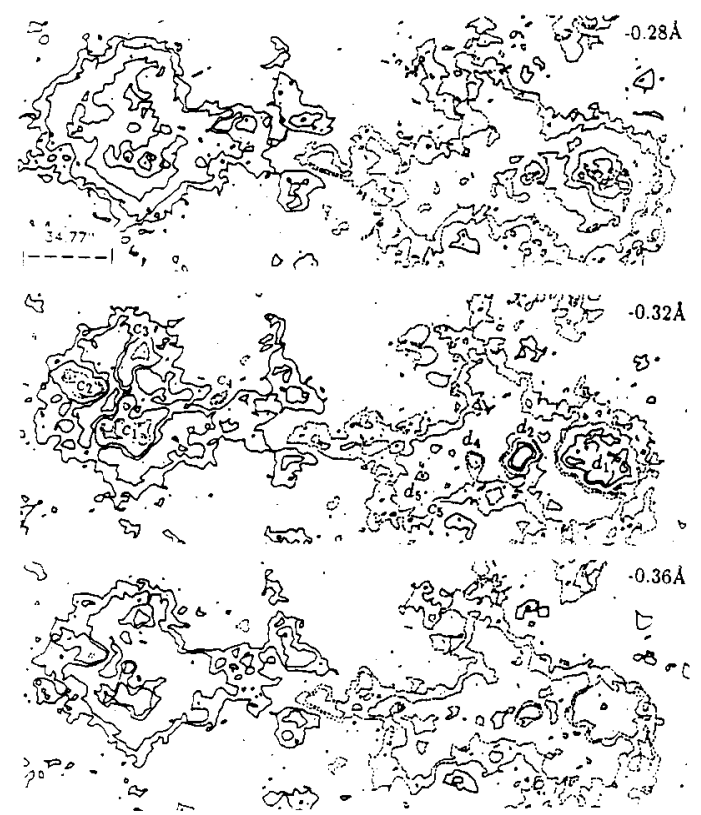

Fig. 1. A series of chromospheric longitudinal magnetograms obtained at different wavelengths in the blue wing of the $\mathrm{H} \beta$ line in AR 7117 on 1992 March 30 . The solid (dashed) contours correspond to positive (negative) fields.

\section{REVERSED POLARITY CHROMOSPHERIC MAGNETIC STRUCTURES}

Recently, attention has been drawn to differences between chromospheric and photospheric magnetic fields, especially polarity reversals in active regions (e.g. Livingston 1973; Chen et al. 1989; Zhang 1991; Wang and Shi 1992). Li Wei (1992) analyzed more than 300 similar chromospheric reversed structures and distinguished four types. Possible explanations of the observed polarity reversal in chromospheric magnetograms are the following:

(a) Twists in the magnetic lines of force in the lower solar atmosphere.

(b) Effects of blending by photospheric lines (e.g. FeI $\lambda 4860.98 \AA$ is close to the general working wavelength of the Huairou magnetograph) in the wing of the $\mathrm{H} \beta$ line.

(c) Effects due to uncompensated Doppler shifts of the chromospheric line.

\section{ANALYSIS OF OBSERVED REVERSED POLARITIES}

1. To determine the influence of photospheric blended lines on the measured chromospheric magnetic field, a series of longitudinal magnetograms at different wavelengths in the blue wing of the $\mathrm{H} \beta$ line around the general working wavelength in active regions was obtained at the Huairou Solar Observing Station (Figure 1). We find that: 


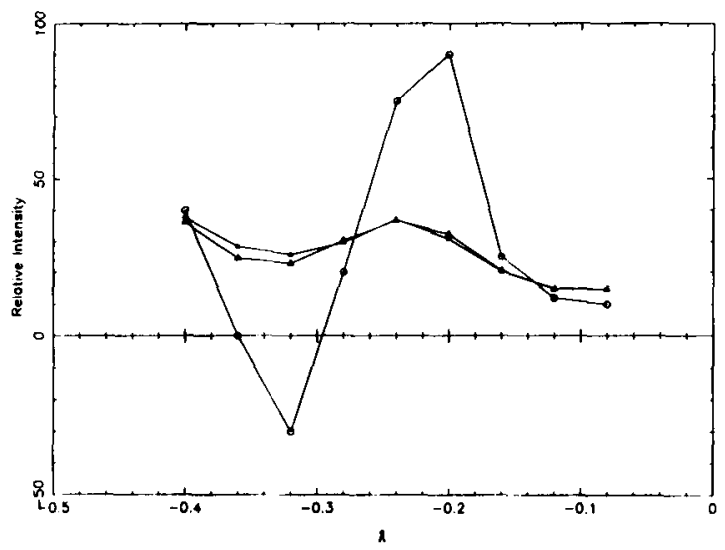

Fig. 2. Averages of magnetic fields in Active Region 7117 on 30 March 1992 measured at different positions in the blue wing of $H \beta$. The circles are averages of umbral fields and show a polarity reversal at $-0.32 \AA$. The triangles are average values outside of umbrae.

(a) Opposite polarity features $\left(c_{1}, c_{2}, c_{3}, d_{1}, d_{2}\right.$ and $\left.d_{4}\right)$ on the chromospheric magnetograms appeared at umbrae at wavelengths between -0.32 and $-0.36 \AA$ from the $\mathrm{H} \beta \lambda 4861.34 \AA$ line center.

(b) On magnetograms at other wavelengths (outside -0.32 to $-0.36 \AA$ from the $\mathrm{H} \beta$ line center), the polarities of the chromospheric magnetograms were the same as the photospheric ones (Figure 2).

2. Profiles of the $\mathrm{H} \beta$ line were obtained in a quiet region, penumbra and umbra of a medium-sized sunspot near the center of the solar disk. The equivalent width of the $\mathrm{H} \beta$ line is $4.2 \AA$ and its effective Landé splitting is about 1. In the weak-field approximation, the Stokes parameter $V$ and $\mathrm{dI} / \mathrm{d} \lambda$ are proportional to each other. This approximation is certainly appropriate for the $\mathrm{H} \beta$ line (Stenflo et al. 1984). This spectral analysis of $\mathrm{H} \beta$ shows the following:

(a) $\mathrm{dI} / \mathrm{d} \lambda$ of the $\mathrm{H} \beta$ line near $-0.3 \AA$ from the $\mathrm{H} \beta$ line center in umbrae shows an opposite sign relative to the neighboring wavelengths (Figure 3 ). This result is similar to that obtained by the Huairou magnetograph.

(b) Reversal of $\mathrm{dI} / \mathrm{d} \lambda$ does not occur at these wavelengths in the blue wing of the $\mathrm{H} \beta$ line in penumbrae and quiet regions.

3. We conclude from the analyses of chromospheric magnetic fields using a series of chromospheric magnetograms at different wavelengths and from the $\mathrm{H} \beta$ line profiles in active regions, the following main results:

(a) In umbrae, the polarity reversals in the chromospheric magnetograms relative to photospheric ones are probably caused by the effects of the blended photospheric line ( $F e I \lambda 4860.98 \AA$ ) in the $\mathrm{H} \beta$ wing.

(b) The separation of the blended photospheric line cores from the $\mathrm{H} \beta$ line near the observing wavelength increases the possibility of a spurious observed polarity reversal due to violent Doppler motions of the chromosphere.

(c) Some chromospheric reversed polarity magnetic structures relative to the photospheric field may exist in solar active regions outside of umbral areas. 


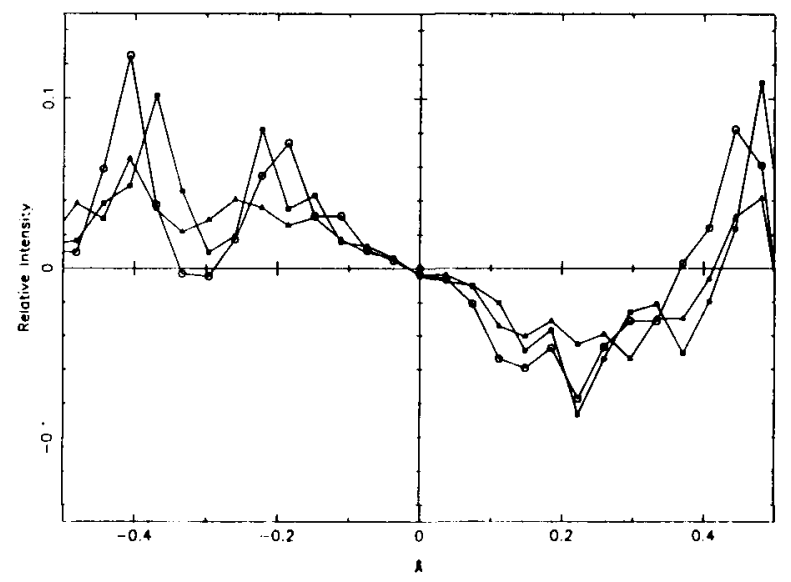

Fig. 3. The function $-d I / d \lambda$ of $H \beta$ line profiles in the quiet sun (triangle), penumbra (square), and umbra (circle).

These are probably caused by twisted lines of force or geometrical perspective effects.

(d) For accurate measurements of the chromospheric magnetic field, a 2-D spectrograph is necessary.

\section{ACKNOWLEDGMENTS}

We wish to thank Prof. C. Fang for help with the $\mathrm{H} \beta$ line profiles. This research was supported by the Chinese Academy of Sciences and National Science Foundation of China.

\section{REFERENCES}

Chen, J., Ai, G., Zhang, H., and Jiang, S. 1989, Suppl. Publ. Yunnan Obser., 108

Li, W. 1992, personal communication

Livingston, W.C. 1973, World Data Center A for Solar-Terrestrial Physics Report Collected Data Report on August 1972 Solar-Terrestrial Events, ed. H.E. Coffey, 95

Stenflo, J.O., Harvey, J.W., Brault, J.W., Solanki, S.K. 1984, A\&A, 131, 333

Wang, J. and Shi, Z. 1992, Acta Astronomica Sinica, 33, 95

Zhang, H. 1991, presented at The First China-Japan Seminar on Solar Physics Workshop, Kunming, China.

Zhang, H., Ai, G., Sakurai, T., and Kurokawa, H. 1991, Solar Phys., 136, 269 